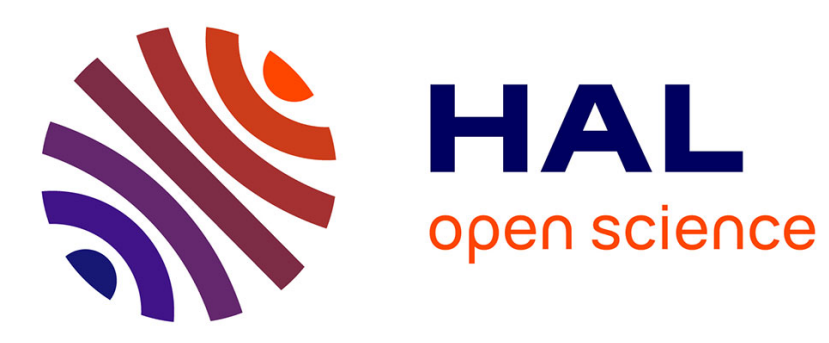

\title{
Télévision à la carte, un divorce annoncé
}

Madeleine Akrich, Cécile Méadel

\section{To cite this version:}

Madeleine Akrich, Cécile Méadel. Télévision à la carte, un divorce annoncé. Réseaux : communication, technologie, société, 2006, 24 (139), pp.75-100. halshs-00174695

\section{HAL Id: halshs-00174695 \\ https://shs.hal.science/halshs-00174695}

Submitted on 19 Jun 2009

HAL is a multi-disciplinary open access archive for the deposit and dissemination of scientific research documents, whether they are published or not. The documents may come from teaching and research institutions in France or abroad, or from public or private research centers.
L'archive ouverte pluridisciplinaire HAL, est destinée au dépôt et à la diffusion de documents scientifiques de niveau recherche, publiés ou non, émanant des établissements d'enseignement et de recherche français ou étrangers, des laboratoires publics ou privés. 


\title{
Télévision à la carte : un divorce annoncé
}

\begin{abstract}
" The current rise of expectations regarding ITV* is not the first in modern history. In 1964, AT\&T demonstrated the 'picture phone' with the promise of interactivity at a distance. During the 1970s several two-way cable TV projects were launched in the US, to be followed by particular two-way cable services such as video-on-demand in the 1980s and full service networks in the 1990s (...). None of these innovations resulted in the breakthrough of ITV. » [van Dijk et de Vos, 2001], p. 461. * Interactive TeleVision
\end{abstract}

La situation française ne fait pas exception à ce constat : depuis les années 80 , différents projets ont vu le jour, ont fait l'objet d'expérimentations et d'analyses ; force est de constater que la télévision dite " interactive » ne s'est pas encore développée de façon significative, ou en tout cas à la hauteur des espérances, très élevées, qui lui étaient à chaque fois associées [Bonte, 1996] [Lafrance et Brouillard, 1996]. Aujourd'hui, cet audiovisuel à la carte trouve un nouveau support sur internet où depuis 2005 se multiplient les sites permettant le téléchargement de contenus, mis en place en particulier par les grandes chaînes de télévision (Canal+, TF1, Arte...) mais aussi les opérateurs des réseaux (Free, TPS, France Télécom)².

Les diffuseurs de télévision ont inscrit explicitement depuis de nombreuses années leurs projets dans une vision qui met ces applications de " télévision interactive $»^{3}$ (pay-per-view ${ }^{4}$ puis vidéo à la demande ${ }^{5}$ ) en position stratégique : elles pourraient reconfigurer radicalement le monde de l'audiovisuel, introduire une rupture dans l'organisation de ses marchés, redéfinir les relations entre les différents acteurs professionnels de ce domaine et leur rôle respectif, transformer la relation du public au medium et aux produits audiovisuels, modifier les habitudes de consommation.

« Avec la VOD, une nouvelle étape est franchie qui risque fort de révolutionner la télévision. La VOD, en effet, transfère la fonction d'agencement (...) aux téléspectateurs, leur donnant ainsi plein pouvoir sur l'emploi du temps. (...) La télévision est donc en passe de devenir un grand jude-box et la notion même de chaîne -en tant qu'assembleur de programmes et maître des horloges- devient obsolète face à cette nouvelle donne ». [Missika, 2006], pp 44-45.

\footnotetext{
${ }^{1}$ Centre de sociologie de l'innovation. Ecole des mines de Paris

${ }^{2}$ voir l'étude commanditée par le CNC, [NPA Conseil, 2006]

${ }^{3}$ Désignée par la Directive européenne Télévisions sans frontières comme les « services non linéaires », autre manière de souligner l'autonomie du spectateur par rapport au service.

${ }^{4}$ Le pay-per-view est un programme payant à un créneau horaire spécifiée : par exemple, la chaîne Multivision de TPS propose différents programmes répétés à différents horaires ; c'est un peu le principe de la séance de cinéma étendu à la télévision.

${ }^{5}$ Les services de vidéo à la demande permettent au consommateur de disposer, moyennant paiement, du programme de son choix - à l'intérieur d'un catalogue - au moment où il le souhaite.
} 
Cette vision s'exprime de manière élaborée, comme un modèle de nouveau marché qui modifie assez radicalement la répartition des rôles entre les acteurs industriels ${ }^{6}$, et se traduit par un certain nombre de dispositifs constitutifs de ce marché : présentation de l'offre, dispositifs de souscription, tarification etc. Malgré les déconvenues du passé, aujourd'hui encore, ces applications sont placées au cœur des stratégies du monde des médias ${ }^{7}$. Pour essayer de comprendre à la fois leur développement limité et les possibilités qui semblent s'entrouvrir aujourd'hui, nous allons revenir sur trois expériences françaises qu'il nous a été donné d'analyser à des époques différentes et avec des objectifs spécifiques en les réexaminant à partir du cadre proposé par « l'économie des qualités » [Callon, et al., 2000]. La première étude menée concernait la conception technique du terminal d'abonné des réseaux de vidéocommunications de $1^{\text {ère }}$ génération [Akrich et Boullier, 1989] dans les années 80; il s'agissait de comprendre la manière dont l'usager était appréhendé dans les processus d'innovation. La seconde, au début des années 90, portait, dans le cadre d'une recherche plus large autour de l'innovation dans les services, sur une expérience de pay-per-view au sein d'un réseau câblé de la banlieue parisienne [Méadel, 1995a]. Enfin la dernière enquête, très récente, s'est attachée à restituer les pratiques effectives des usagers dans le cadre d'un nouveau service de vidéo à la demande proposé sur un réseau de diffusion TV par ADSL.

Cette perspective de « l'économie des qualités » place au cœur de la dynamique des marchés le travail de qualification des produits, travail effectué par une multitude d'acteurs, du producteur au client-usager-consommateur final. En effet, tout marché suppose l'existence de listes de produits « qualifiés », c'est-à-dire aux caractéristiques plus ou moins stabilisées, produits entre lesquels se porte le choix des clients. De ce fait, la compétition s'exerce sur cet espace de qualification; sans arrêt, les acteurs travaillent sur la redéfinition du couple produit / consommateur, en utilisant les ressources de la technique, comme du marketing, de la publicité, du design, etc. Ils cherchent à produire de nouvelles qualifications qui ont pour effet de singulariser certains produits, d'en « disqualifier » d'autres, et ce faisant d'en détacher ou d'y attacher certains consommateurs. Cette logique de singularisation se trouve aujourd'hui poussée à son maximum, et en conséquence rendue très visible, au travers de l'utilisation des technologies de l'information et de la communication : des portails, aux comparateurs divers en passant par les blogs, ou encore les forums, les recueils d'avis, on ne compte plus les dispositifs susceptibles de produire des différences toujours plus fines dans un espace de produits sans cesse élargi.

Cette question de la singularisation est centrale dans le cas des applications qui nous intéressent : elle se manifeste à deux niveaux différents. Il s'agit tout d'abord de singulariser

\footnotetext{
${ }^{6}$ « De multiples manières, la télévision interactive peut être considérée comme une réponse industrielle à la convergence des technologies de communication, un nouveau paradigme lancé par les technologies numériques ». [Sawhney, 2002]

${ }^{7} \mathrm{Cf}$ « La VOD va entraîner des « transformations majeures dans le mode d'accès aux programmes et modifier l'économie du secteur, notamment le financement de la production », souligne l'étude NPA. Un peu à l'image des bouleversements entraînés par l'arrivée des DVD dans les années 1990. » dans " La vidéo à la demande bouscule les relations cinéma-télévision », Le Monde, 7 avril 2006. Sur les quatre premiers mois de 1'année 2006, sept articles du Monde évoquent la vidéo à la demande : notons cependant qu'aujourd'hui, la vidéo à la demande est investie d'un pouvoir décisif non seulement dans le secteur télévisuel mais aussi et peut-être davantage dans la concurrence entre fournisseurs d'accès Internet.
} 
certaines offres de services par rapport aux seuls « bouquets » en y ajoutant le pay-per-view ou la vidéo à la demande (VOD). En second lieu, il s'agit aussi de singulariser chaque prestation, c'est-à-dire de positionner de manière potentiellement avantageuse chaque achat de télévision à la carte dans un espace de choix pour le consommateur. Ces deux niveaux sont évidemment complémentaires et liés : à moyen terme, ces services ne peuvent constituer un avantage décisif pour les opérateurs-diffuseurs de programmes que si les consommateurs se laissent convaincre de façon récurrente par l'offre de prestations qui leur est faite. Or, cette prestation de service s'appuie sur un produit audiovisuel pré-existant, éventuellement accessible par d'autres intermédiaires : chaînes de télévision, cinéma, DVD ou cassettes vidéo... Selon que l'on considère cette disponibilité comme un gage de réussite - comment le consommateur refuserait-il de disposer au moindre effort et à un coût raisonnable de ce qu'il apprécie dans des conditions souvent moins confortables ? - ou comme une concurrence sérieuse, les applications de télévision interactive ne vont pas être positionnées de la même manière dans la stratégie des opérateurs, ni susciter les mêmes types d'approches.

Dans une première partie, nous reviendrons sur chacun des projets en décrivant la place qu'occupent la VOD ou le pay-per-view dans la stratégie d'ensemble développée et en analysant le travail spécifique effectué par les opérateurs en vue d'aider le client éventuel à repérer, identifier, positionner cette prestation de manière à ce qu'elle prenne sens, puisse se singulariser, et donner lieu à une réalisation. Nous verrons que, si le pay-per-view ou la vidéo à la demande y apparaissent à chaque fois comme des leviers puissants de transformation des marchés de l'audiovisuel, capables d'étendre et de diversifier le spectre des qualifications possibles pour certains produits, la question des moyens nécessaires pour convaincre le consommateur a été abordée de manière différente ; c'est essentiellement le projet le plus récent qui a donné lieu à une élaboration fine de dispositifs permettant aux usagers d'appréhender, de qualifier, d'évaluer les produits qui leur sont proposés et donc de les engager éventuellement dans un processus de transformation de leurs habitudes, goûts etc.

Puis, dans une seconde partie, nous confronterons la relation entre produits et consommateurs, telle qu'elle a été inscrite dans les dispositifs des réseaux ; on constatera en particulier que la singularisation de la prestation est loin d'être achevée : les biens autour desquels le marché est supposé se construire ont un contour flou, ils sont ambigus, polysémiques, de par la multiplicité des références auxquels ils sont renvoyés, par le service lui-même et par la diversité des pratiques des usagers. Pris dans un entrelacs de relations qui brouillent son identification, la VOD peine à se constituer en tant que prestation bien définie. D'un certain point de vue, elle est sans cesse désingularisée.

Enfin, en conclusion, nous reviendrons sur la notion d'interactivité, et nous nous interrogerons sur les différentes significations qui lui sont accordées dans le domaine télévisuel. Nous nous demanderons si la vidéo à la demande qui a longtemps été considérée comme une application minimale mais prodromique de la télévision interactive n'est pas appelée à se développer en dehors d'elle, via Internet. Assiste-t-on à la fin de la télévision, comme le soutiennent certains analystes ? Ou à la fin du téléviseur ? Ou encore à la multiplication des formes de consommation de programmes audiovisuels ? [Missika, 2006] 


\section{LA « TELEVISION INTERACTIVE ॥ COMME DEVELOPPEMENT STRATEGIQUE}

Au début des années 80 , le $\mathrm{CNET}^{8}$ plante un décor, qui préfigure les évolutions ultérieures, au travers des réseaux de vidéocommunications de première génération (RV1G). L'utilisation d'un nouveau matériau, la fibre optique, constitue le cœur autour duquel s'organise la définition de ces réseaux. Cette fibre optique présente deux caractéristiques intéressantes : elle permet le transport simultané de signaux hétérogènes (télévision, son hifi, données, téléphone, etc) avec un niveau de qualité jusqu'alors inégalée par les autres supports existants (câble coaxial par exemple) ; elle autorise des échanges d'informations dans les deux sens entre tous les points du réseau et réalise en quelque sorte le mariage entre le câble téléphonique traditionnel et le câble coaxial. Se trouve ainsi ménagée la possibilité de ce que l'on appelle dès cette époque une interactivité du réseau.

Cette interactivité peut être valorisée de trois façons. Tout d'abord, elle permet d'introduire une différenciation dans les modes de taxation des services, en transférant certaines pratiques du monde de la téléphonie à celui de l'audiovisuel : au delà de l'abonnement classique, des taxations à la durée et au programme, du type pay-per-view, peuvent être envisagées. En second lieu, elle permet d'installer des dispositifs d'observation et de mesure du trafic qui bouleversent les méthodes habituelles utilisées pour le calcul des audiences. Enfin, elle rend possible la mise en place de codes, composés par l'utilisateur, qui permettent de définir un accès sélectif à certains services.

Dans l'esprit des concepteurs, ce réseau se présente comme une sorte de réseau universel qui rendra obsolètes un certain nombre de produits ou de services : vu d'aujourd'hui, il ressemble furieusement à l'ADSL qui permet à la fois l'accès à Internet, au téléphone, à la télévision. Dès cette époque, différentes applications sont envisagées comme le pay-per-view, ou, à plus long terme, la vidéo à la demande. Certaines formes sophistiquées sont même imaginées qui tirent parti au maximum des possibilités techniques comme la diffusion d'émissions d'information proposées par les laboratoires pharmaceutiques à destination exclusive des médecins et qui leur seraient accessibles au moment qui leur convient : dans cette configuration, les opérateurs de réseau vendraient non seulement le droit de diffusion sélective des émissions, mais aussi des données sur l'écoute (nombre de spectateurs, durée d'écoute etc.) qui permettrent aux laboratoires de mesurer l'impact de leur politique de communication. Dans le même mouvement, les opérateurs de réseau donnent la main aux usagers qui deviennent en partie leurs propres programmateurs et échappent ainsi en partie aux contraintes temporelles imposées par les diffuseurs.

Le RV1G vise donc à l'instauration d'une nouvelle donne dans le domaine audiovisuel, en produisant une différenciation dans les modes de partage des coûts et des bénéfices entre les acteurs (usagers, annonceurs, fournisseurs de programmes, gestionnaires des réseaux), différenciation accompagnée d'une redéfinition des services rendus. Cette innovation qui est pensée comme une innovation totale - reconfiguration radicale du marché en même temps que mise en place de technologies nouvelles - se développe cependant essentiellement sur le plan technique : il s'agit de construire une machine parfaite, tellement parfaite qu'au fond la

\footnotetext{
${ }^{8}$ Centre National d'Etudes des Télécommunications
} 
question de la singularisation trouve son aboutissement dans la conception technique. Au point que les concepteurs ignorent purement et simplement les attachements existants des consommateurs à certains dispositifs ou certaines pratiques : pourquoi s'embarrasser du problème complexe posé par le raccordement du magnétoscope au système, puisque le RV1G fournira à terme tous les contenus désirables, avec une qualité d'image inégalée [Akrich, 1990], et que, par ailleurs, les fournisseurs de programmes ne pourront résister à une plateforme qui leur permettra de vendre des contenus, en leur donnant de surcroît l'accès à des informations précises sur les consommateurs ${ }^{9}$ ? Un travail conséquent des promoteurs du réseau va donc porter sur l'interface de transaction : comment permettre que ces transactions « inévitables » puissent avoir lieu de façon efficace et en toute sécurité ?

L'expérience tournera court cependant : les fournisseurs de contenus ne se laissent pas séduire par ce dispositif et les abonnés de l'expérience pilote ne se laissent pas détacher du magnétoscope récemment arrivé sur le marché français. L'opérateur technique échoue à se positionner en médiateur / organisateur des marchés de l'audiovisuel.

La seconde expérimentation semble a priori plus limitée. En 1993, un opérateur de réseau câblé propose pendant trois mois du pay-per-view : un programme, principalement des films pendant cette expérimentation, est diffusé à heures fixes sur un canal spécialement affecté et le client a seulement le choix de l'acheter ou de ne pas l'acheter. Ce câblo-opérateur est associé à France-Telecom - gestionnaire technique du réseau câblé - qui fournit un terminal, le Visiopass, mis au point par un laboratoire du CNET, destiné à assurer le débrouillage des programmes achetés et, pour ceux des abonnés qui sont dotés du modèle sophistiqué, la transaction. Mais derrière la modestie apparente de l'expérience, ses promoteurs ne cachent pas les ambitions dont il s'agit d'éprouver le réalisme : les services en pay-per-view sont les chevau-légers du redéploiement du câble, dont le marché peine alors à se développer, et de son insertion dans l'univers du multimédia. Ils permettent de faire entrer chez l'usager et de tester un décodeur, le Visiopass qui pourra servir de support pour bien d'autres services que des programmes payants : du téléachat, des services à public ciblé (comme la bourse), de la haute définition, de la radio haute qualité, de la télématique...[Méadel, 1995b] avec en ligne de mire, pour les concepteurs, l'intégration des trois dispositifs de communication, téléphone, informatique et télévision. Par ailleurs, tout comme dans le cas des RV1G et plus généralement de ce type de projet [Sawhney, 2002], l'expérience par câble redéfinit les

\footnotetext{
${ }^{9}$ On retrouve de nos jours une stratégie formellement assez semblable autour du TiVo, sorte d'enregistreur / disque dur intelligent. Les gestionnaires de réseaux comme les fournisseurs de contenus sont a priori réticents par rapport aux systèmes d'enregistrement qui permettent contourner le paiement des droits de diffusion, droit d'auteur etc. Cette question prend une acuité particulière dans le cas du numérique qui, couplé à l'ordinateur, permet l'échange à coût quasi-nul. «L'astuce » de TiVo, c'est d'avoir couplé le dispositif d'enregistrement à la transmission de données aux gestionnaires de réseaux et aux fournisseurs de contenus ; peut se développer une autre approche de l'audience 1) basée sur les consommations réelles et la structure de la consommation pour chaque foyer (même idée que dans RV1G) 2) qui permet de contourner les difficultés posées par le fait que les dispositifs d'enregistrement brouillent les cartes (les usagers échappent en partie au « contrôle ») et perturbent la « valorisation » des temps publicitaires. [Carlson, 2006] Cependant ce modèle est fragile : les publicitaires commencent à refuser de payer pour des spectateurs qui soit ne visionnent jamais les programmes enregistrés, soit utilisent l'avance rapide pour sauter les publicités. (« Bar the Door. TV Ads Want Your TiVo. », D. Leonhardt, New York Times, 17 mai 2006)
}

http://www.nytimes.com/2006/05/17/business/media/17leonhardt.html) 
relations qu'entretiennent les différents acteurs de la diffusion, distributeur, producteur de programmes, opérateur et client. L'éditeur - qui développe un système de télévision en payper-view - n'achète plus le programme, il lui ouvre des cases horaires qui peuvent éventuellement lui donner accès à un public et il partage le produit de la vente avec le producteur et l'opérateur. Ainsi se débarrasse-il des frais de programmes en les faisant supporter par les producteurs et les clients.

Dans cette configuration, l'application de télévision interactive, qui est supposée dotée d'un pouvoir d'attraction du consommateur en raison de ses qualités intrinsèques, sert d'appât afin de transformer le consommateur, de l'attacher au décodeur et à des formes de transaction nouvelles. Durant l'expérimentation, deux éléments importants apparaissent ; tout d'abord, la nécessité d'équiper le consommateur afin qu'il puisse avoir une certaine visibilité de l'offre : comment en effet l'attirer vers ces nouveaux contenus qui ne figurent dans aucune grille générale de programme et ne se manifestent que sous la forme d'un canal particulier affecté à cet effet, crypté tant que l'usager n'a pas acheté le programme? D'où le développement d'un journal mensuel de programmes, d'annonces en clair, y compris sur le canal mosaïque, tous efforts qui se traduisent par des coûts fixes que le système devait minimiser. En second lieu, l'expérimentation montre que les abonnés qui disposent d'un Visiopass dit interactif ont consommé dix fois plus que ceux qui devaient passer commande par l'intermédiaire du minitel ou du téléphone, même si la commande était exécutée quasi-immédiatement : autrement dit, c'est bien la prestation de service dans son ensemble qu'il faut envisager si l'on veut comprendre la dynamique de ce type d'applications, prestation qui resurgit ici dans toute son épaisseur. À l'évidence et la transparence supposées par les RV1G - le consommateur sait ce qu'il veut, et l'interface est un simple intermédiaire technique qui rend possible l'exercice de cette volonté - l'expérience de pay-per-view vient opposer un démenti clair : le poste de télévision est d'abord une boîte opaque et les modalités par lesquelles peut s'exprimer la demande sont constitutives de cette demande.

Ici, encore, ce qui a été conçu comme une expérimentation tourne court, alors que les câbloopérateurs sont engagés dans une phase de restructuration industrielle. Canal + , qui a le vent en poupe, est alors présenté comme un obstacle insurmontable : la chaîne contrôle la diffusion des films en première exclusivité dont les opérateurs du câble considèrent qu'ils sont un élément indispensable pour arriver à séduire le consommateur.

Dans l'expérience la plus récente et sans doute la plus aboutie, l'application de télévision interactive est encore placée en position d'appât : elle est supposée faire la différence entre les offres des fournisseurs de bouquet. Cette expérience concerne une offre de télévision par ADSL qui a été lancée au tout début de l'année 2004. Les opérateurs de réseau ont entamé des négociations avec un des deux fournisseurs majeurs de bouquet, puis avec le second, de manière à pouvoir proposer aux clients un système qui soit à parité avec l'existant du point de vue des chaînes disponibles, et se singularise par la disponibilité de ce nouveau service de VOD. Celui-ci est défini comme un moyen inédit d'accéder à des contenus disponibles par ailleurs, mais aussi à des contenus peu, mal ou pas diffusés par la télévision. Pour de multiples raisons, l'opérateur va donc chercher à développer des partenariats avec des distributeurs de court-métrage, de films anciens, de documentaires, etc. La télévision par ADSL se trouve d'emblée en tension entre deux définitions : d'un côté, elle peut apparaître comme un nouveau mode de diffusion des « bouquets », et c'est au travers des bouquets de 
chaînes, de leur notoriété, de leur pouvoir d'attraction que se fait l'adhésion pour des clients qui, pour une raison ou une autre, n'avaient pas accès aux modes "classiques " (câble ou parabole) de diffusion. De l'autre, elle préfigure la télévision de demain, "interactive » comme il se doit, et l'opérateur de réseau devient alors un peu le chef d'orchestre de cette mutation en développant des applications et en "offrant » des espaces de déploiement à d'autres acteurs, comme les fournisseurs de bouquets.

Cette tension est très palpable dans les premiers mois de commercialisation du service : alors qu'une part de la communication en direction des médias se fait autour de la VOD considérée comme innovante, dans les espaces de vente le « bouquet » est placé au premier plan, grâce en particulier au matériel promotionnel mis à disposition par le diffuseur. Elle se traduit aussi par l'existence au démarrage du service de deux contrats souscrits par l'abonné - un premier contrat avec l'opérateur de réseau qui donne l'accès matériel au réseau Télévision par ADSL et au service de VOD, un second avec le fournisseur de bouquet pour l'accès aux chaînes - et par une organisation de l'interface qui sépare d'entrée de jeu deux espaces, celui du bouquet et celui de la VOD.

Dans ce contexte, on comprend que le travail visant à équiper l'abonné afin qu'il souscrive à une prestation VOD soit cantonné à « l'intérieur » du service et que la mise en perspective de cette offre particulière dans l'ensemble de ce qui est proposé par l'intermédiaire de l'ADSL soit laissée à l'initiative de l'abonné. Ce travail de singularisation se porte sur trois composantes principales : tout d'abord, sur le choix des contenus qui, comme souligné précédemment, ouvre, pour une part du catalogue, vers des programmes peu médiatisés et peu présents sur les grands supports de diffusion, cinéma, télévision hertzienne. En second lieu, se pose la question des modalités de présentation des programmes ; le cinéma va constituer ici le modèle de référence. En effet, trois supports vont être repris ou élaborés pour les programmes jusqu'alors peu médiatisés : une « affiche » (vignette visuelle représentant le programme), un texte résumé de présentation, et une «bande-annonce » qui, pour les programmes qui n'en disposent pas au départ, est constituée généralement d'un extrait du programme. Le troisième volet de travail, la mise en perspective de l'ensemble des programmes, pose des problèmes redoutables, car l'écran de télévision et la télécommande offrent des possibilités limitées en termes de densité de contenu et de modalités de circulation entre les contenus. Le modèle choisi est un modèle arborescent qui, tout d'abord, sépare les contenus en deux grandes catégories, cinéma et télévision, chacune divisée en sous-catégories. Chaque grande catégorie dispose d'un écran sur le pourtour duquel sont disposées ces sous-catégories; le centre de l'écran est occupé par une " nouveauté » mise en avant. La sélection d'une de ces souscatégories ouvre à nouveau sur un écran qui présente un programme " phare » et permet d'avoir accès à la liste des autres programmes de la catégorie, puis éventuellement de visionner la bande-annonce, de lire le texte de présentation, et d'acheter un programme. Ce dispositif de présentation va être complété, quelques mois après le lancement du service et à l'instar de ce qui avait été mis en place dans l'expérience de pay-per-view, par un journal d'une dizaine de pages présentant à la fois le service et un échantillon de programmes.

Les trois expériences de « télévision à la carte » que nous venons de décrire s'inscrivent dans une même perspective, le développement de services multimédia faisant l'objet de transactions individualisées. On note cependant au fil du temps un resserrement des ambitions : dans les deux premiers cas, ces services de VOD et de pay-per-view n'étaient 
qu'un exemple parmi bien d'autres des services qui devaient se mettre en place grâce aux réseaux. S'appuyant sur un produit et des pratiques supposés banals, ils jouaient le rôle « d'avant-garde » préfigurant et testant des configurations à venir. Sans doute, le développement d'Internet a-t-il en grande partie rempli les objectifs qui étaient fixés aux réseaux multimédia, non sans avoir introduit dans le jeu une multitude de nouveaux acteurs. Reste la VOD qui, de par son affinité historique avec la télévision - jusqu'à récemment, seuls les réseaux câblés de télévision étaient en mesure techniquement de diffuser ce type de programme - se trouve à nouveau élevée au rang d'application interactive modèle pour la télévision. Cependant, le développement encore limité de la VOD laisse ouverte la question suivante : à quelles conditions peut-on transformer le téléspectateur en téléacheteur de programmes audiovisuels? Nous venons de voir comment les acteurs du développement de la VOD dans l'expérience la plus récente ont travaillé sur la présentation de l'offre. Nous allons maintenant nous tourner vers les abonnés au service pour analyser la manière dont les cadrages proposés par les concepteurs sont appréhendés.

\section{LES USAGERS OU L'IMPOSSIBILITE DE STABILISER UNE QUALIFICATION POUR LA PRESTATION}

Les résultats que nous présentons dans la suite sont issus d'une enquête basée sur une cinquantaine d'entretiens approfondis avec des abonnés au service de télévision par ADSL, qui ont déjà acheté des programmes en VOD. Au cours de ces entretiens, nous avons cherché à resituer l'usage de la VOD des usagers dans l'ensemble des pratiques de consommation audiovisuelle et à analyser spécifiquement ce que met en jeu l'utilisation du système : pour ce faire, l'entretien s'est accompagné de manipulations qui visaient à saisir la manière dont l'offre se présentait dans l'interaction à l'usager.

Le service VOD propose une multitude de prises, certaines ont été disposées à dessein, d'autres sont en quelque sorte inscrites dans les « dispositifs en contexte ", c'est-à-dire au travers des routines de manipulation et de consommation qui se sont créées au fil du temps. D'autres prises sont ménagées par le système TV dans son ensemble qui peuvent entrer en concurrence avec celles, spécifiques, de la VOD. C'est à l'analyse des relations entre ces divers éléments et de leurs effets sur le consommateur que nous allons nous consacrer dans la suite.

\section{LA VOD DANS L'ENSEMBLE DU SERVICE}

De façon peu surprenante, compte tenu des stratégies de vente que nous avons décrites précédemment, la VOD n'est pratiquement jamais un élément déterminant dans la décision d'abonnement. La différenciation du service TV par ADSL s'opère bien davantage par le mode de raccordement, la prise téléphonique, que par tout autre critère. A l'époque du lancement, un autre opérateur Internet avait déjà inclus la télévision dans son offre ; la stratégie suivie dans notre projet est différente puisqu'elle détache l'offre télévisuelle de l'internet, voulant se donner les moyens de toucher une clientèle peu technophile qui ne souhaitait pas forcément avoir accès à Internet. L'espace des comparaisons est donc assez clairement défini et perçu comme étant celui des abonnements télévisuels ; l'offre en question se veut similaire aux autres sur le plan des contenus - le même bouquet que sur le câble et le 
satellite - et singulière du point de vue technique, avec une installation moins contraignante puisqu'elle passe par le réseau téléphonique.

Peu visible au départ, la VOD reste un élément assez mal identifié, même quand elle a fait l'objet d'une utilisation. Le service parle peu au client : la documentation promotionnelle qui est envoyée pour présenter les produits est rare et peu appréciée. Le petit journal pour les abonnés ne rend compte que d'une partie du choix disponible. Et le service lui-même ne fait pas ou très peu sa propre promotion, à la différence des chaînes de flux qui ne cessent de s'annoncer pour attirer l'attention et provoquer le désir.

« La vendeuse n'était pas trop au courant. Le nouveau produit arrivait dans ma région. [La VOD] ne m'intéressait pas au moment où je l'ai souscrit, c'était surtout pour avoir TPS. Une fois que le produit m'est arrivé, je me suis dit “tiens c'est un vidéo club”. »

« On ne connaît pas. C'est vrai qu'on ne reçoit rien de l'opérateur, rien, aucune info. Donc, peut-être qu'on passe à côté de connaissances. (...) Je ne savais pas qu'il y avait des choses spécifiques, à la carte. Nous on a eu des infos que de TPS. »

La présence sur le bouquet d'un service de pay-per-view accentue la confusion : sur une chaîne, des programmes, films, événements sportifs ou culturels sont proposés en diffusion multiple à horaires fixes ; qualifiés tous deux de «à la carte », le pay-per-view et la VOD ne sont pas clairement séparés dans l'esprit des usagers. La confusion est renforcée par le fait que les contenus proposés peuvent être identiques et être programmés par ailleurs sur les chaînes du bouquet. La pratique du « versionnage » et sa différenciation des prix [Bomsel, et al., 2006], de plus en plus développée dans le domaine audiovisuel, prend une allure quelque peu surréelle aux yeux des consommateurs ; le rapprochement effectué par le medium suscite au sens propre la constitution d'un espace commun et donc la possibilité d'une comparaison : or cette comparaison peine à déboucher sur une correspondance entre les variations de prix observées et une représentation claire des différences entre prestations.

«Récemment il ont mis « People - Jet Set 2 », que je pouvais également avoir sur [le service de pay-per view]. (C'est le même prix ?) Non c'est 5,6€ et c'est $5 €$ sur l'autre et c'est exactement le même film...»

« Le film « Les 11 commandements » que j'ai pris au vidéo-club à 3,15€, sur le service de [VOD] il est à 10 tickets soit 5 Euros. Dans le même temps [le service de pay-per view].le faisait à 2 ou $3 € \ldots$ Maintenant aller payer pour voir un reportage qui est passé sur Arte, pfou (soupir) c'est un peu fou. Je vais pas payer 3 ou $4 €$ un reportage qui est déjà passé sur Arte. Surtout si le reportage m'intéresse, généralement on ne passe pas à travers. On l'a regardé ou on l'enregistre. »

D'autant que certaines différences, qui tiennent à l'organisation des marchés, et aux rapports de force entre les différents acteurs, restent largement incompréhensibles : pourquoi serait-il possible d'enregistrer un film diffusé sur une chaîne, ou en pay-per-view, et impossible d'enregistrer le même film diffusé en VOD ... alors même qu'il en a coûté plus cher à l'usager?

« Le gros désintérêt, c'est qu'on paye et on ne peut pas les enregistrer. On paye quelque chose qu'on ne peut pas avoir. C'est une horreur absolue. On le regarde et on sait que dès qu'on a fini de regarder, on ne peut plus s'en servir. J'achète en DVD, je peux m'en servir autant de fois que je veux. Je peux même le prêter, faire un échange, je ne sais pas... A mes amis, on en discute... Pour discuter, il faut pouvoir le voir, or si on ne peut pas le voir... Je ne vois pas l'intérêt. »

Cette confusion est renforcée par le fait que le prix des produits en VOD n'a pas de rapport avec la prestation technique, mais apparaît lié indissolublement au produit : cela rend alors 
difficile de comprendre pourquoi un contenu libre d'accès à un moment va être payant à un autre moment. Du coup, à l'incertitude inhérente à l'industrie des contenus dont les consommateurs ne peuvent apprécier la valeur qu'après consommation [Bomsel, et al., 2006], se rajoute une seconde incertitude sur le prix liée à l'ambiguïté, rendue manifeste par nombreuses protestations concernant l'enregistrement, sur ce qui fait l'objet de la transaction : que paie-t-on ? Une prestation ou un contenu ? Une séance de vidéo à la demande ou un film ?

Enfin, le service de TV sur ADSL incorpore un autre modèle de rétribution, celui de l'abonnement, c'est-à-dire un paiement forfaitaire en échange d'un accès illimité à des contenus. La comparaison entre prix de l'abonnement et prix d'un programme en VOD donne un peu le vertige à certains consommateurs aux moyens modestes : comment en effet imaginer que cinq films puissent représenter la même dépense qu'un abonnement mensuel ${ }^{10}$ ?

Les références contenues dans le fonctionnement du réseau conduisent donc à un brouillage : la nature de la prestation n'apparaît pas clairement ; plus encore, la variabilité du prix dont la rationalité n'est pas accessible au consommateur devient un élément de description du produit, produisant une forte incertitude sur la valeur même de ce qui est acheté.

\section{Un eVENTAIL DE PRATIQUES DE CHOIX...}

On voit donc que les prises offertes par le service aident peu le téléspectateur à se forger une définition stabilisée de la VOD insérée dans une offre de télévision de type « traditionnel ». Comment dans ses interactions pratiques avec le dispositif, le téléspectateur s'en débrouille-til ? L'ample littérature qui cherche à rendre pensable l'activité du téléspectateur [Le Grignou, 2003] insiste à la fois sur ses capacités d'appropriation et d'autonomisation par rapport au dispositif mais aussi sur l'extrême diversité des pratiques, des agencements mis en œuvre et des compétences mobilisées [Jouët, 2000], contre une approche ordinaire du dispositif présenté comme fortement prescripteur.

L'arbitrage du téléspectateur ne se fait pas entre chaînes du bouquet et VOD ou pay-per-view, mais entre programmes ; or ceux-ci sont proposés dans une quantité telle que la comparaison et le choix deviennent difficiles, jusqu'à alimenter des formes de rejet, même chez les gros consommateurs que sont les abonnés du service étudié :

«Le problème si ça ne plait pas, c'est que, comme on sait qu'on a plein de chaînes ailleurs, on va zapper, aller ailleurs et c'est là qu'on est perdu »

« Ils pourraient mettre 800 chaînes, des fois il n’y a rien. Rien, rien qui procure de l'intérêt. C'est très subjectif et personnel. (...) Ce n'est pas facile... Il y a quand même énormément de séries B. C'est mauvais quoi. On ne supporte pas, donc on ne regarde pas. Après,

\footnotetext{
10 « Je prends le panel de base, il a un coût. Il est évident que toutes leurs chaînes à option c'est pas moi qui vais y souscrire. C'est quand je vois des films à 5-6€ ou un match de foot ou n'importe quoi, peu importe, c'est toujours 5-6, 7 ou $8 €$, ça me fait sourire. ». Dans une micro-enquête norvégienne [Ling, et al., 1999] sur une expérience de VOD en 1995-96, concernant 17 familles, les usagers avaient exprimé spontanément des idées autour du modèle économique : dans un couple, l'un des deux soutenait que le prix devait être analogue à celui de la location d'une cassette, alors que l'autre défendait un modèle ouvert dans lequel la VOD devient une source libre d'information via une redevance globale. Or dans le cas qui nous occupe, il faut à la fois payer une redevance globale qui donne l'accès au service et payer chaque prestation individuelle.
} 
il y a aussi beaucoup de déjà vu. Il y a à voir une seule chaîne. Moi, je ne suis pas du genre à regarder dix fois le même film... On fait autre chose. »

Les stratégies d'arbitrage jouent des outils ordinaires du choix en matière de télévision : magazines de programmes, presse généraliste, journal du bouquet. Mais l'abondance des programmes, que les téléspectateurs perçoivent comme forte redondance ${ }^{11}$, perturbe les routines traditionnelles et les transforme, dans la mesure où la synthèse devient impossible.

«Avant, vous achetiez votre Télé 7 Jours. Vous regardiez dans la semaine ce qu'il y avait. Tiens "mardi on pourra regarder ça". Et là vous ne pouvez pas le faire. Tous les soirs, je vais regarder vite fait ce qu'il y a sur les chaînes. Parce qu'il y a tellement de films qu'on n'arrive pas à... mis à part si j'en vois un qui me plaît vraiment mais autrement, demain je suis incapable de dire ce que je vais regarder. J'achète mon journal, je le regarde vite fait, mais on peut pas se souvenir. Il y a trop d'offres... Il y a plusieurs chaînes le soir qui vont me proposer du film. On a du mal à suivre. »

Les pratiques d'exploration peuvent être planifiées, avec analyse attentive des programmes, repérage « en stabilobossant », programmation des visionnages, ou être laissées à l'imprévu de la préférence d'un moment ; elles peuvent être fixes et s'organiser toujours à peu près selon le même modèle ou au contraire varier selon le moment, les spectateurs présents, les envies, l'offre connue ; elles peuvent viser une exploration variée et diversifiée ou se limiter aux canaux familiers. Les consommateurs n'optent pas pour un seul de ces formats et oscillent avec des pondérations différentes entre l'organisé et le spontané, le stable et l'instable, la routine et l'aventure, la mémoire de ce que l'on a prévu de regarder et le hasard du zapping... La forme prise par l'arbitrage varie en fonction des moments de la journée, de la composition du public, mais aussi des programmes eux-mêmes, avec des routines stabilisées, au moins pour une période donnée, comme les informations quotidiennes (où l'on « est » encore largement TF1, France 2 ou LCI), ou les programmes de flux récurrents comme les feuilletons.

« On connaît [les programmes] par cœur. C'est-à-dire que l'on a des séries récurrentes que l'on regarde habituellement, donc on connaît les jours. Mais sinon, non, on ne regarde pas trop à l'avance. Moi, je suis quand même assez... je zappe... Je dois reconnaître que je zappe et je découvre un peu comme ça. »

Le bouquet de chaînes ajoute son propre dispositif de choix à ceux dont dispose le téléspectateur : la mosaïque, cette chaîne qui montre sur un même écran les images en direct d'une série de chaînes et permet de les sélectionner sans passer par le long détour de leur numéro. Ce dispositif prescrit des formes de choix : à l'intérieur du bouquet, la mosaïque exclue certaines chaînes qu'elle ne propose pas à l'affichage (et qui ne peuvent donc être sélectionnées qu'en tapant le numéro sur la télécommande); elle hiérarchise les services à la fois par son ordre de présentation des chaînes et en exigeant plusieurs manipulations pour s'en extraire et parvenir, par exemple, à la VOD ; elle privilégie un repérage par l'image en direct. Elle permet un double visionnage qui suspend le choix ou du moins le laisse ouvert, tout comme le fait aussi le zapping :

« Ça m'arrive de regarder la mosaïque et des fois je regarde chaîne après chaîne. (C'est commode ?) Oui. On reste sur la même chaîne et en même temps on regarde ce qu'il y a sur

\footnotetext{
${ }^{11}$ Ce qui a été montré par les économistes : la multiplication du nombre de chaînes accroît la redondance plus que la variété des contenus et n'augmente pas nécessairement sur les chances d'optimiser sa satisfaction. [Neuendorf, et al., 2000]
} 
d'autres chaînes. (Une image en grand et une petite image dedans ?) Oui, il y a un rectangle qui est au milieu, et, en même temps, on regarde tout ce qui se passe. »

Zapping, comme mosaïque laissent le dernier mot au choix qui, pour reprendre les termes d'un téléspectateur, "saute aux yeux ». On remarque que même les téléspectateurs qui ont une consommation très planifiée, très organisée et usent de multiples instruments de différenciation des produits accordent au zapping ou à mosaïque, voire aux deux combinés, un rôle central dans le choix.

Ainsi, confrontés à une multiplicité de programmes qui ne peuvent être embrassés dans leur totalité mais qui ont aussi un caractère répétitif, et à un acte de choisir non moins récursif, les consommateurs mettent en place différentes routines de choix. Certains font de l'exploration méthodique des chaînes puis se décident en bout de course après avoir vu un grand nombre de possibilités. D'autres ont un ordre particulier de sélection des programmes et vont s'arrêter au premier programme jugé intéressant... D'autres encore commencent par le connu (par exemple les chaînes habituellement fréquentées) et ne se résolvent à d'autres explorations que si celui-ci ne les satisfait pas. Cette variabilité des façons de faire touche aussi à la manière de consommer le programme : on note ainsi des comportements fréquents de réévaluation des choix en cours de visualisation, et, comme l'a montré Carrie Heeter [Heeter, 1985], cette réévaluation est d'autant importante qu'il s'agit de programmes payants, par exemple ceux proposés sur les chaînes nécessitant une souscription particulière en sus de l'abonnement de base, comme s'il fallait sans arrêt contrôler la qualité de ce que l'on paie de manière spécifique. Comment s'insère le choix de la VOD dans cette pluralité de stratégies?

\section{... PEU INTEGREES DANS L'INTERFACE}

Les modes de choix « spontanés » que nous venons de décrire sont faiblement articulés avec la VOD qui, du point de vue de l'interface, s'approche davantage du modèle Minitel que de celui des bouquets télévisuels. Cette interface ne reprend pas les dispositifs sur lesquels s'appuient les choix des téléspectateurs : il n'y a pas de pages de type mosaïque qui montreraient en même temps toute l'offre de VOD, voire, comme le fait celle du bouquet, les programmes réputés les plus appréciés. Par ailleurs, la télécommande ne permet pas un accès instantané à l'image : pour avoir une image d'un programme en VOD, il faut en passer par une procédure en plusieurs étapes, avec à chaque fois les tâtonnements d'un arbre de décision.

L'interface de l'offre est conçue, on l'a vu, pour permettre une mise en valeur des contenus et favoriser l'adéquation entre contenus et téléspectateurs. Or, les téléspectateurs jugent le dispositif du service que nous avons étudié fastidieux et peu éclairant. Cette appréciation nous semble moins liée à une ergonomie défaillante qu'au format du catalogue mis en télévision. Le passage par l'écran de télévision et son interface, la télécommande, l'absence de clavier, l'organisation hiérarchique de l'offre contraignent les formats de déplacement dans le catalogue. Comme le choix est présenté de manière séquentielle, le consommateur doit faire un arbitrage à chaque niveau pour accéder au niveau suivant ; il n'y a pas de passerelle possible et en cas d'erreur, le téléspectateur doit accomplir à nouveau tout le parcours pour parvenir à la bonne case. L'autre entrée possible, par la fonction recherche, n'est guère plus appréciée, car elle opère par l'intermédiaire de listes ou menus déroulants peu parlants. Enfin, la réactivité du dispositif est faible, si faible que le téléspectateur peut penser à tort qu'il a bloqué le système. 
« Là vous avez toutes les rubriques... Sinon vous pouvez chercher par nom, mais ce n'est pas évident. J'ai déjà essayé. La fonction recherche est un peu longue. Quand on va dans les rubriques, on est obligé de visualiser toute la liste, c'est un peu long. Et on ne peut pas passer d'une page à l'autre directement. Ça c'est dommage »

« Je n'ai pas vraiment la visibilité de ce qu'il y a comme programme. Quand on veut voir ce qui est proposé, passer par un système de liste où on voit pas beaucoup de choses à la fois et les délais de réponse sont des fois un peu long pour passer d'une page à l'autre... C'est un peu énervant... » « Il y a plein de thèmes, il y a plein de trucs à regarder. C'est long. A chaque fois qu'on zappe, au fur à mesure qu'on avance dans les programmes, pour revenir en arrière on ne peut pas mettre un numéro de page. Par exemple, pour revenir à la page précédente, faut retourner, retourner...»

Le téléspectateur décide d'abord s'il veut de la « télé » ou du « ciné » dans un partage qui peut lui poser problème : certains consommateurs considérent que les programmes télévisés sont de l'ordre du gratuit, d'autres n'imaginent pas y retrouver des documentaires, etc. ; puis, à l'intérieur de chacun de ces deux grands espaces, il opte pour une catégorie dans un ensemble hétérogène, qui met à équivalence un genre (comédie, théâtre...), un public (enfant dans l'une, jeunesse dans l'autre), un format (court-métrage), voire une qualification au registre incertain (passion, aventure...). Il peut échapper aux sous-catégories en choisissant les « nouveautés », mais il risque alors d'être trompé sur le calendrier de référence, le service parlant de lui (les derniers programmes mis à l'affiche en VOD) tandis que le téléspectateur entend bien souvent calendrier du cinéma, avec les sorties en salle puis en DVD..., d'où une intense déception devant une offre vue comme par un consommateur comme « paléolithique ».

Une fois qu'il s'est situé dans cette catégorisation et qu'il a repéré un programme possible, le consommateur de VOD peut regarder son affiche et lire les brèves informations fournies. S'y ajoute un dispositif spécifique au service VOD qui lui permet de « savoir vraiment de quoi parle » le produit proposé : la bande annonce lui donne un bref aperçu du programme, un peu à la manière de la mosaïque, mais il a dû accomplir un long périple pour parvenir à ce stade là et n'a donc pas retrouvé l'instantanéité et la rapidité qu'il mobilise ordinairement face à son téléviseur.

«Ce qui est pas mal, c’est les bandes annonces. Ça c'est super. Vous voyez tout de suite. L'inconvénient, c'est que, quand vous êtes parti sur une bande annonce, vous ne pouvez pas l'arrêter. Apparemment il n'y a pas de moyen de dire 'j' ai vu merci'. Vous avez la bande annonce jusqu'au bout. Mais c'est bien. Ça donne une idée sur un film que vous ne connaissez pas. »

« On peut regarder la bande annonce, voir ce que c'est comme film. On ne va pas se faire arnaquer en donnant $5 €$. C'est vrai qu'on a peur d'être déçu, alors on regarde avant. »

Face à la souplesse et à la labilité de la consommation d'une chaîne de télévision où le choix, les comportements sont en permanence révisables, le consommateur de VOD est mis en situation de choisir avec une capacité de retour moindre, le paiement constituant un engagement qui limite les changements en cours de programme (d'autant plus qu'il ne peut pas, dans le dispositif conçu dans cette offre, le mettre de côté pour le consommer ultérieurement, ce que permettent les autres dispositifs).

Ce parcours complexe invite en fait le consommateur à passer de l'offre fortement organisée par le flux, la programmation, les genres, les habitudes... que lui propose la télévision à une offre par produit où il est laissé très libre y compris pour en construire la lisibilité. Pour s'orienter dans le bouquet, le téléspectateur dispose de différents prescripteurs/ facilitateurs, et 
peut passer sans coup férir de l'un à l'autre, du journal de programmes à la mosaïque, de la mosaïque à la télécommande : en dépit de ce qui est perçu comme une surabondance de l'offre, l'ensemble de ces dispositifs permet d'éviter la paralysie de l'âne de Buridan ; à chaque instant, une prise se présente au télespectateur qui permet de contourner ou de résoudre en pratique la question du choix. Pour la VOD, malgré l'existence de dispositifs d'aide au choix - résumé, bande-annonce - il se trouve en un sens plus démuni : il n'a pas les appuis socio-cognitifs que fournissent les dispositifs matériels habituels [Cochoy, 1999], ni ceux, plus récents, que proposent les « pairs » habilement mis en scène par les sites de vente sur internet (« les internautes qui ont aimé/ acheté/ choisi X ont aussi aimé/ acheté/ choisi, W, $\mathrm{Y}, \mathrm{Z} »)$. Il manque donc quelques intermédiaires qui donneraient du sens à la liberté de choix présentée comme un argument fort de la VOD : en effet, " les dispositifs d'aide au choix, comme toutes les normes sociales ou comme tous les cadres techniques nous libèrent d'un harassant travail de définition et de cadrage de nos rapports et de nos interactions et [ainsi,] sont [...] beaucoup plus 'émancipateurs' qu'ils ne sont 'contraignants'. » ([Cochoy, 2002] pp 153-154). Ici, le consommateur potentiel est bien laissé libre face à ses choix, mais sans l'équipement qui permet de sortir de l'embarras de cette ouverture ${ }^{12}$. La singularisation des produits à l'intérieur même du service de VOD s'avère donc délicate, et cette difficulté retentit sur le service en général qui, du coup, peine à trouver sa place par rapport au bouquet. Certes, il apparaît comme différent, mais cette différence est plutôt perçue comme négative. Nous nous trouvons ici dans une configuration de second ordre, très généralisée (grandes surfaces, agences de voyage, vente sur internet...), dans laquelle la concurrence porte en partie sur des formes d'organisation du marché : la singularisation de chaque service ou prestataire est liée à sa capacité à équiper de manière adéquate le consommateur dans sa démarche de choix, et donc à construire pour chacun la singularité des produits vers lesquels il se porte. ${ }^{13}$

\section{LA VOD DANS L'enSEMBLe DES PROdUITS ET SERVICES AUDIOVISUELS}

Pour le moment, nous avons envisagé la VOD principalement à l'intérieur de l'univers lié à la télévision. Mais de par sa constitution même, la VOD offre des prises diverses à l'interprétation, que saisissent les consommateurs en l'inscrivant dans divers formats de consommation de produits audiovisuels, de la télévision « ordinaire » ou par bouquet, au cinéma, en passant par l'achat ou la location de supports, etc. Certains inscrivent la VOD dans une concurrence avec le cinéma, mettant en balance la commodité d'accès, l'immédiateté de la consommation, les avantages et inconvénients des salles... Pour partager un certain nombre de repères (qui interviennent dans le choix unitaire d'un produit), le rapprochement pointe la problématique spécification des programmes en VOD qui ne bénéficient pas -ou très indirectement- de la médiatisation propre aux films, avec son agenda, ses prescripteurs, ses

\footnotetext{
${ }^{12}$ De façon significative, la consommation de VOD n'a été significative que pour des produits pour lesquels il n'y avait aucune incertitude ni du côté de l'offre, ni du côté de la demande, à savoir des séries télé pour enfants déjà diffusées et doncconnues - LuckyLuke, Titeuf - et proposées à des prix très modiques.

${ }^{13}$ Ce qui ne veut évidemment pas dire qu'il y aurait une césure entre d'un côté l'organisation du marché, et de l'autre, les produits eux-mêmes et la manière dont leurs qualités ont été mises en forme par des dispositifs extérieurs : l'organisation du marché inclut les produits dans leur épaisseur propre et c'est ce qui complexifie quelque peu l'analyse.
} 
lieux connotés (par exemple salle d'arts et d'essai / multiplex). D'autres comparent la VOD avec le commerce de DVD ou cassettes vidéo ${ }^{14}$, avec les mêmes avantages du point de vue de la mobilité, mais un choix beaucoup moins large et une carence en matière de prescripteurs (vendeurs, promotion, affichage, autres clients...). Sans compter qu'il est difficile d'enregistrer et que la fonction patrimoniale que remplit pour certains l'achat d'un support n'est pas ici satisfaite. Cette fluctuation entre différents espaces de définition du service rend les pratiques complexes et hésitantes.

Le rapprochement avec ces formes de consommation audiovisuelle est devenu patent lorsque le service est passé d'un système d'achat par tickets, achetés en bloc, avec une tarification des programmes en nombre d'unité, à un paiement à l'acte, avec prix en euros. Le prix du ticket était certes connu, mais n'était mobilisé qu'au moment d'un achat global de tickets, qui pouvait être découplé de toute consommation. On se trouvait donc plutôt dans une logique de type abonnement / consommation de biens déjà achetés. Lorsque le prix du produit est rendu visible à chaque transaction, on passe dans une logique d'achat qui est plus exigeante en termes de justification et de qualification ; chaque décision fait l'objet d'un arbitrage qui met le programme visé directement en concurrence avec les offres que le consommateur estime comparables :

« Ils ont gagné un peu en visibilité parce qu'avant c'était une histoire de tickets. On achetait 8 tickets, on se rendait pas trop compte combien ça vous coûtait. Maintenant ils ont mis ça en Euros ce qui fait que c'est visible tout de suite. Quand j'ai vu les tarifs, c'est plus cher que mon vidéo club. Surtout le vidéo club il est juste en bas de chez moi.»

Confrontés à ce nouveau service, le consommateur est donc dans le flou : dans le flou d'un produit avec une signalétique et des modes d'accès faibles, et dont la valeur est incertaine, l'interface opaque, le catalogue mal apprécié, obscur. De manière assez significative, les deux principales vertus de la VOD mises en avant par les promoteurs du service (disponibilité des contenus et liberté des horaires) sont invoquées par un nombre limité de consommateurs, dont les profils sont atypiques : par exemple ceux qui travaillent pendant les moments forts de la programmation des chaînes ou qui ont des contraintes familiales particulières. Pour une large majorité des interviewés, la VOD peine à trouver sa place et s'insère de manière mouvante dans les pratiques télévisuelles des spectateurs ; les consommations sont limitées et le jugement à l'égard du service plutôt réservé. Les consommateurs de VOD sont donc confrontés à une prestation non stabilisée, dans un univers peu mis en forme. Ils ont certes acquis un pouvoir de décision sur le choix du contenu et la temporalité de la programmation, mais sans que les moyens fournis ne leur permettent d'équiper leur jugement et de faciliter leur parcours.

\section{Conclusion}

Face à la supposée passivité du téléspectateur [Boullier, 2004], les dispositifs de VOD semblaient ouvrir une alternative, une brèche apte à le rendre plus autonome, libre de ses choix, de son temps. Les trois projets étudiés font de la télévision à la carte, VOD comme

\footnotetext{
${ }^{14}$ Rapprochement qui avait déjà été fait par les pionniers norvégiens de Videotorg [Ling, et al., 1999], confrontés à un système de VOD encore très expérimental dont nul, y compris les concepteurs, n'avait une claire représentation.
} 
pay-per-view, une application phare qui doit reconfigurer le monde industriel de l'audiovisuel et changer les formes de consommation : elle apparait emblématique des possibilités offertes par la télévision dite « interactive ».

Comment analyser justement cette interactivité ? Van Dijk en propose une définition à partir de quatre niveaux interdépendants : le multilatéralisme de l'interaction qui doit a minima se produire dans deux directions ; le caractère synchrone de l'interaction et la répartition des temps d'intervention des différentes parties ; le contrôle du processus d'interaction et enfin la capacité des parties à comprendre l'environnement de l'interaction [van Dijk et de Vos, 2001]. Ce cadre qui permet de classer tout type d'échange situe à un niveau assez faible la télévision dite interactive en général et les projets que nous avons étudiés en particulier ${ }^{15}:$ le multilatéralisme est limité à deux parties ; les temps d'interaction sont asymétriques, étant très largement monopolisés par le diffuseur ; le téléspectateur contrôle bien le processus d'interaction mais dans le cadre prédéfini qui lui est proposé et enfin la capacité à comprendre les conditions de l'interaction est faible tant du côté des publics que de celui des diffuseurs. Nos trois projets développent donc des dispositifs faiblement interactifs, avec peu de capacité d'échanges, une asymétrie des communications et une faible interconnaissance, l'offre ayant peu de remontées de la demande et la demande une vision limitée de l'offre ${ }^{16}$.

Cette interactivité limitée semble constitutive du dispositif technique « télévision »: de Vos [de Vos, 2001] a montré, au terme d'une vaste enquête auprès des experts professionnels, comment ceux-ci peinaient à sortir de cette définition pauvre de l'interactivité, contraints qu'ils sont par l'objet et les formes d'usage qui se sont cristallisées autour de lui. Or, nous avons vu tout au long de notre analyse comment le travail de singularisation des programmes proposés en VOD était handicapé par la faiblesse de l'interactivité et la lourdeur des procédures d'exploration de l'offre qu'elle implique. Ceci est devenu d'autant plus sensible pour le consommateur que se sont développés sur internet, ces dernières années, des dispositifs riches, souples, fluides permettant une navigation « marchande » autrement plus convaincante, y compris pour des produits audiovisuels.

On peut alors se demander si les services de distribution de programmes à la carte ne sont pas plutôt appelés à se développer en dehors de la télévision et en premier lieu sur internet qui offre des capacités d'interactions largement plus développées que ce qui a été exploré dans les différents projets de VOD ou de pay-per-view [Berendt, 2002] [Eronen, 2004], surtout si l'on est attentif à la diversité des acteurs qui proposent désormais ce type d'offre, opérateurs de télévision ou de télécommunications, mais aussi libraires en ligne, syndicats de professionnels indépendants, centres d'archives, particuliers... L'interaction peut y être multilatérale, avec possibilité de faire intervenir d'autres acteurs, par exemple des clients ou des intermédiaires

\footnotetext{
${ }^{15}$ De ce point de vue, cette analyse est convergente avec celle de Kim \& Sawhney qui mettent en évidence la contradiction entre la structure traditionnelle de la communication télévisée (où le médium est censé faire face à des publics inertes) et l'interactivité supposée de la télévision moderne [Kim et Sawhney, 2002]

${ }^{16}$ Les échanges sont circonscrits à trois types d'informations : des données sur le programme choisi puis consommé, ce qui permet d'établir une taxation à l'unité ou à la durée ; des données d'audience prélevées automatiquement, sans la participation de l'usager; enfin sont communiquées par les usagers des données sur la différenciation des membres du foyer qui permet, par exemple par des codes, de limiter l'accès à certains programmes ou le volume de la consommation.
} 
de l'offre (comme les critiques). Le consommateur a une capacité de contrôle de l'échange plus importante dans la mesure où il est situé dans un espace de choix plus ouvert avec une offre déclinée en une pluralité de versions (streaming / téléchargement, achat/location...), les formats d'ordonnancement peuvent permettre des tris multiples, par prix, par ordre alphabétique, par jugement, par catégories (les nouveautés/les derniers jours, les catégories par genre/catégories par public, etc.). Ces services peuvent aussi être en situation d'explorer de multiples dispositifs de captation des préférences et des comportements (cf les listes de préférences, les offres jointes, les avis de consommateurs...). Autrement dit, l'espace de qualification des produits que propose internet est autrement plus équipé que celui des services VOD liés à la télévision; il dispose une offre beaucoup plus large et en même temps beaucoup plus facilement différenciable, c'est-à-dire qu'il permet infiniment mieux de singulariser les produits. Par ailleurs, la diffusion par chaînes, que propose la télévision et vers laquelle toute son organisation technique converge, " aspire » le téléspectateur et empêche tout attachement durable à la VOD. Pour toutes ces raisons, on peut faire l'hypothèse que la migration de la distribution individuelle de programme vers internet est inéluctable.

Pour autant et contre un certain nombre de prophéties, il ne s'agit pas de condamner la télévision et d'entrevoir sa mort, là où l'on n'a qu'une mutation de plus d'un service sans cesse chahuté depuis son origine tant dans ses technologies que dans la construction de son marché et de son public. Sans doute assiste-t-on à une diversification des formats de consommation de l'audiovisuel, mais on peut faire l'hypothèse qu'il y aura cohabitation entre les différents services plus que substitution. Enfin, il ne faudrait pas non plus opposer la richesse interactive d'internet à la pauvreté interactive de la télévision. Le choix du contenu et du moment de visualisation n'est qu'une facette possible de l'interactivité [Raudaskoski, 2002] qui, dans la télévision traditionnelle, s'exprime sans doute ailleurs, par exemple dans la capacité des programmes à permettre des interactions entre usagers.

\section{Biblographie}

Akrich, M., 1990, "De la sociologie des techniques à une sociologie des usages: l'impossible intégration du magnétoscope dans les réseaux câblés de première génération", Techniques et Culture, 16, pp 83-110.

Akrich, M. et Boullier, D., 1989, Le K, monographie n¹, in Boullier D. (ed.), Genèse des modes d'emploi : la mise en scène de l'utilisateur final, LARES-CCETT.

Berendt, A., 2002, "The changing landscape for multi access portals", Business Information Review, 19, 2, pp 21-29, http://bir.sagepub.com/cgi/content/abstract/19/2/21.

Bomsel, O., Geffroy, A. G. et Blanc, G. L., 2006, When Internet meets Entertainment. The Economics of Digital Media Industries, Paris, Presses de l'Ecole des Mines.

Bonte, J., 1996, La télévision à la carte aux Etats-Unis, Paris, PUF.

Boullier, D., 2004, La télévision telle qu'on la parle. Trois études ethnométhodologiques, Rennes, L'Harmattan.

Callon, M., Méadel, C. et Rabeharisoa, V., 2000, “L'économie des qualités", Politix, 13, 52, pp 211-239. 
Carlson, M., 2006, "Tapping into TiVo: Digital video recorders and the transition from schedules to surveillance in television", New Media Society, 8, 1, pp 97-115, http://nms.sagepub.com/cgi/content/abstract/8/1/97.

Cochoy, F., 1999, "De l'embarras du choix au conditionnement du marché. Vers une socioéconomie de la décision", Cahiers internationaux de sociologie, 106, juillet, pp 145-173.

Cochoy, F., 2002, Une sociologie du packaging ou l'âne de Buridan face au marché, Paris, Presses universitaires de France.

de Vos, L., 2001, Searching for the Holy Grail. Images of interactive television, European Media Masters of Arts, Utrecht, University of Utrecht.

Eronen, L., 2004, User centered desing of new and novel products : case digital television, Helsinki, Helsinki University of Technology.

Heeter, c., 1985, "Program selection with abundance of choice. A process model", Human Communication Research, 12, 1, pp 126-152.

Jouët, J., 2000, "Retour critique sur la sociologie des usages; Communiquer a l'ere des réseaux", Reseaux: Issy les Moulineaux, 100, pp 487-521.

Kim, P. et Sawhney, H., 2002, "A machine-like new medium: A theoretical examination of interactive TV", Media, Culture \& Society, 24, pp 217-233.

Lafrance, J.-P. et Brouillard, P., 1996, "À la conquête électronique de la toison d'or ou la mise en place des contenus et des services sur les autoroutes de l'information", Réseaux, 78.

Le Grignou, B., 2003, Du côté du public. Usages et réceptions de la télévision, Paris, Economica.

Ling, R. L., Nilsen, S. et Granhaug, S., 1999, "The domestication of video-on-demand: folk understanding of a new technology", New Media Society, 1, 1, pp 83-100, http://nms.sagepub.com/cgi/content/abstract/1/1/83.

Méadel, C., 1995a, "L'innovation mise à l'épreuve. Une expérience de télévision payante", In al., A. H. (ed), Lyonnaise des Eaux-Dumez. Une entreprise face à ses innovations : politique, technique, environnement, Centre de Sociologie de l'Innovation, Ecole des Mines.

Méadel, C., 1995b, “Le D2Mac ou les revers de la compatibilité”, Quaderni, 26, été, pp 99112.

Missika, J. L., 2006, La Fin de la télévision, Paris, Seuil, La République des idées.

Neuendorf, K., Jeffres, L. W. et Atkin, D., 2000, "The television of abundance arrives: cable choices and interest maximization", Telematics and Informatics, 17, 3.

NPA Conseil, 2006, Le développement de la vidéo à la demande en France et en Europe, Paris, CNC.

Raudaskoski, P. L., 2002, Interactivity as it happens : Interactions at and with computer and television media, Aalborg, DK, Aalborg University , Department of Communication and Psychology.

Sawhney, H., 2002, "A machine-like new medium: A theoretical examination of interactive TV”, Media, Culture \& Society, 24, 217-233.

van Dijk, Jan A. et de Vos, L., 2001, "Searching for the Holy Grail. Images of interactive television", New Media \& Society, 3, 4, pp 443-465. 
